\title{
Cell-mediated immunity predicts the probability of local recruitment in nestling blue tits
}

\author{
M. CICHOŃ \& A. DUBIEC \\ Institute of Environmental Sciences, Jagiellonian University Gronostajowa 7, Kraków, Poland
}

Keywords:

brood size manipulation;

fledging success;

immune response;

local recruitment;

selection.

\begin{abstract}
We investigated whether the variation in T-cell-mediated immune function of blue tit nestlings affected their fledgling success and the probability of local survival. We studied the relationship between immune function and survival under two rearing conditions: control, unmanipulated, and experimentally enlarged broods. Brood enlargement had negative effects on nestling immune response. Immune response was positively related to fledgling success and it predicted the probability of local recruitment. However, the relationship between immune response and the probability of recruitment was significantly positive only among control broods and nonsignificant among enlarged broods. The effect of immune response on the recruitment probability was not affected by variation in body mass. Our study suggests that selection for immune responsiveness seems to be weak or even absent under unfavourable rearing conditions as simulated by brood size enlargement. Therefore, year-toyear environmental variation and environmental heterogeneity may constrain evolution towards higher immune responsiveness.
\end{abstract}

\section{Introduction}

As immune function evolved to protect individuals from pathogens and endogenous aberrations of cell functions (Roitt et al., 1996) it should strongly affect prospects of survival and future reproduction. Therefore, variation in immune function should be closely linked to fitness. In consequence, immune function has recently become a central focus for research on the evolution of life history strategies and sexual selection (e.g. Zuk \& Stoehr, 2002). An increasing number of studies seem to suggest that variation in immunity makes sexual ornaments reliable signals of individual condition (e.g. McGraw \& Ardia, 2003; Peters et al., 2004) and that immune function trades for limited resources with other vital physiological functions (Lochmiller \& Deerenberg, 2000; Norris \& Evans, 2000). This leads to the conclusion that immune function is a physiological trait undergoing individual optimisation. A number of studies show that immunity is affected by environmental conditions and that it has a considerable heritable component (e.g. Saino et al., 1997;

Correspondence: M. Cichoń, Institute of Environmental Sciences,

Jagiellonian University Gronostajowa 7, 30-387 Kraków, Poland

E-mail: cichon@eko.uj.edu.pl

Present address: A. Dubiec, Institute for Ornithology, Polish Academy of

Sciences Nadwiślańska 108, 80-680 Gdańsk, Poland.
Brinkhof et al., 1999). Therefore, variation in immune function may constitute an important selective target, if this variation does indeed translate into fitness differences. However, the adaptive significance of the immune system is more often assumed than actually demonstrated: there is still little evidence of whether variation in immune function has any fitness consequences in vertebrates (Norris \& Evans, 2000; but see review by SchmidHempel, 2003, for some evidence in insects). Only few studies have shown a positive correlation between individual immune function and the probability of survival in nestling birds (Christe et al., 1998; Nordling, 1998; González et al., 1999; Christe et al., 2001; see Møller \& Saino, 2004 for review). Recently, Råberg \& Stjernman (2003) reported a significant directional and stabilizing selection operating through differential survival on humoral immune responsiveness against two different antigens in adult blue tits. Meta-analyses of 12 studies on survival in relation to nonspecific immune response to a challenge with an antigen or other measures of immune function demonstrated that the mean effect size adjusted for sample size was relatively high (Møller \& Saino, 2004). This study showed that immune response accounted for over $18 \%$ of variation in survival.

In the present study, we investigate whether the variation in $\mathrm{T}$-cell-mediated immune function affects 
fledging success and the probability of being recruited to the local population, which is a good estimate of local survival, in blue tit (Parus caeruleus) nestlings. We study the relationship between immune function and survival under two rearing conditions: in natural (control) and experimentally enlarged broods. Since the development of immune function is nutrition dependent (e.g. Gershwin et al., 1985; Klasing, 1998), the alteration of nutritional conditions due to brood size manipulation should affect the development of nestling immune system and this in turn should translate into differences in survival probability. Recent studies have shown that nestlings from naturally large or experimentally enlarged broods, presumably experiencing poor rearing conditions, have lower T-cell-mediated immune response than nestlings from small or experimentally reduced broods (Saino et al., 1997; Hõrak et al., 1999; Saino et al., 2003). We predicted that the relationship between the level of immune response and survival might differ between nestlings from unmanipulated and enlarged broods.

The probability of long-term survival in nestlings of altricial birds is usually attributed to body mass at fledgling (e.g. Tinbergen \& Boerlijst, 1990; Perrins \& McCleery, 2001; see also Merilä et al., 1999, for a significant effect of body condition) and body mass positively correlates with immune response (e.g. Tella et al., 2000a; Westneat et al., 2004). In consequence, any effects of variation in immune function could in fact be explained by variation in body mass. Therefore, we additionally analysed variation in survival probability in relation to immune responsiveness while accounting for the potential confounding effect of body mass. We found that nestling survival and probability of local recruitment were positively related to T-cell-mediated immune response.

\section{Methods}

The experiment was conducted on nest-box breeding blue tits in deciduous woodlands in southern Gotland (SE Sweden, $57^{\circ} 10^{\prime} \mathrm{N}, 18^{\circ} 20^{\prime} \mathrm{E}$ ) in 2002 . The study area covers ca. $33 \mathrm{~km}^{2}$ in total and consists of 15 plots varying in size from 3 to 64 ha separated by arable lands or heaths (for description of the study area see Pärt $\delta$ Gustafsson, 1989). From the end of April, nest boxes were regularly visited to record laying date, clutch size, hatching date and number of hatchlings. In order to create two different rearing environments some broods were subject to brood size enlargement, while the others were left nonmanipulated. In enlarged broods, three randomly selected nestlings were transferred from a donor brood of the same hatching date on day 2 posthatching. This constitutes ca. 30\% increase in brood size. These extra nestlings served only to increase within-nest competition and were not used in the analyses. Control and enlarged broods were matched in terms of brood size ( \pm 1 nestling) and equal hatching date. In total, 25 broods were enlarged and 25 broods constituted a control group, however, three enlarged broods were deserted shortly after manipulation.

T-cell-mediated immune function of nestlings was assessed as a response to phytohaemagglutinin (PHA), which is a standard test used in avian studies (Lochmiller et al., 1993; Brinkhof et al., 1999; Tella et al., 2000a,b). Phytohaemagglutinin is a bean extract, which has a mitogenic effect on T-lymphocytes and its inoculation stimulates dense accumulation of lymphocytes (Goto et al., 1978). When nestlings were 11 days old (day $0=$ hatching day), $0.2 \mathrm{mg}$ of PHA suspended in $0.04 \mathrm{~mL}$ of physiological saline solution was inoculated in the middle of the right wing web (Smits et al., 1999). The thickness of the wing web was measured with a pressure sensitive spessimeter (Mitutoyo, SM-12) prior to and $24 \mathrm{~h}$ after the PHA injection with an accuracy of $0.01 \mathrm{~mm}$. Each measurement was taken three times and as it was highly repeatable (repeatability: prior to injection: $r=0.98, F_{480,962}=190.33, P<0.0001$; postinjection: $\quad r=0.99, \quad F_{480,962}=594.78, \quad P<0.0001$; Lessells \& Boag, 1987) the mean value was used in the further analyses. The level of immune response was calculated as a difference between mean wing web thickness prior to and after the injection.

Nestlings were ringed when 11 -day old and on day 14 post-hatching they were weighed with a Pesola spring balance to the nearest $0.1 \mathrm{~g}$. All nests were inspected a few days after the expected fledging to record fledging success. All adults breeding in our nest-box population were captured in the following two years after the experiment (2003 and 2004) to assess the local recruitment rate. We assume that the majority of the recruiting birds bred in the nest boxes. Breeding attempts in natural holes are rare (own observations). The recruitment rate observed in our population recruitment rate is relatively high in comparison with other blue tit populations.

To study whether brood enlargement affects nestling immune response we performed GLM analyses with a degree of brood size manipulation as a fixed factor and nest identity nested in brood size manipulation. To analyse the determinants of fledging success and the probability of local recruitment, generalised linear mixed models (GLMMs) with a logit link function assuming a binomial (error) variance was run in SAS v8 (SAS Institute Inc., 2000; see Krackow \& Tkadlec, 2001, for details of the procedure). In this analysis, brood size manipulation was introduced as a fixed factor and the level of nestling cell-mediated immune response as a covariate. Since the relationship between immune response and survival may stem from the positive effect of body mass on both immune function and survival, nestling body mass measured 14 days after hatching was additionally introduced as a covariate. Nest identity (ID) was also introduced as a random effect, which allows an estimation of the fixed effects and interaction parameters while accounting for random variation in fledging 
success and the probability of recruitment between broods. First, the full models were run and then nonsignificant interactions $(P>0.4)$ were sequentially removed. Since the relationship between immune responsiveness and the probability of nestling survival and local recruitment may appear to be nonlinear, if, for instance, immune response undergoes stabilizing selection, we additionally tested for nonlinearity introducing a quadratic term of immune response to the above models. However, the models including a quadratic term appeared nonsignificant, so these analyses are not reported in the Results section.

\section{Results}

Brood enlargement had a negative effect on nestling immune response (GLM accounting for within-nest variation; $\left.F_{1,45}=12.94, P<0.001\right)$. Nestling survival until fledging was relatively high and did not differ significantly between control and enlarged broods $\left(\right.$ GLMM; $F_{1,53.5}=0.15$, n.s.): five of 269 nestlings from control broods died before fledging (1.9\%) and seven of 240 from enlarged broods $(2.9 \%)$. However, the probability of successful fledging was positively related to cellmediated immunity in both experimental groups (GLMM; $F_{1,453}=92.9, P<0.001$, Fig. 1$)$.

Twenty nine of 497 fledglings $(5.8 \%)$ were recruited to the breeding population in the following years. The probability of local survival was significantly higher among control broods $(6.4 \%$ vs. $5.1 \%$ in enlarged broods), but it was not related to the level of cellmediated immune response nor to the fledgling body mass (Table 1). However, there was a significant interaction between brood size manipulation and immune response (Table 1), which indicates that the

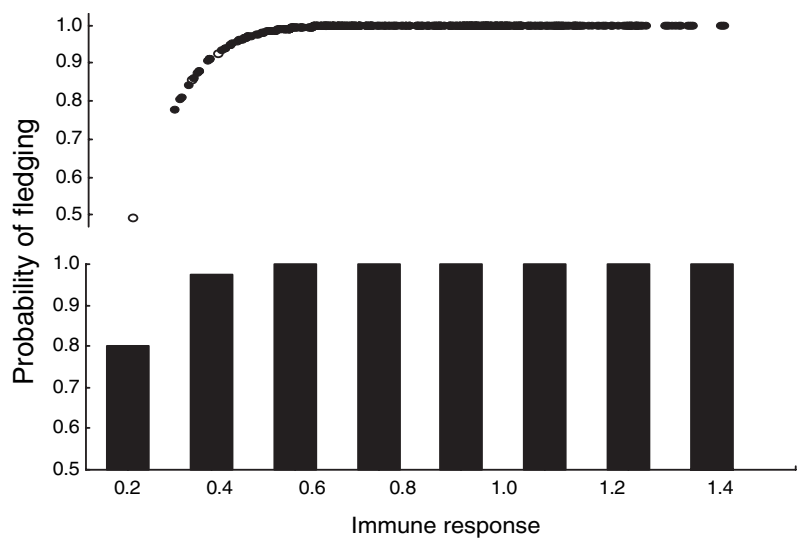

Fig. 1 The relationship between nestling immune response and the probability of fledging. Upper graph presents predicted probabilities derived from logistic regression. Filled circles represents surviving nestlings while unfilled nonsurviving nestlings. The histogram shows proportions of surviving nestlings.
Table 1 The results of generalized linear mixed model of the probability of local recruitment with brood size manipulation (control or enlarged broods) as a fixed factor and cell-mediatedimmune response and body mass at day 14 post-hatching introduced as covariates.

\begin{tabular}{|c|c|c|c|}
\hline & $F$ & d.f. & $P$ \\
\hline Brood size manipulation & 4.54 & 1, 469 & 0.034 \\
\hline Immune response & 0.45 & 1, 469 & 0.50 \\
\hline Body mass at day 14 & 0.08 & 1,469 & 0.78 \\
\hline \multirow{2}{*}{$\begin{array}{l}\text { Brood size manipulation* } \\
\text { immune response }\end{array}$} & 4.99 & 1,469 & 0.026 \\
\hline & Estimate \pm SE & $Z$ & \\
\hline Nest ID & $0.99 \pm 0.1$ & 16.23 & $<0.001$ \\
\hline
\end{tabular}

Nest ID accounts for random variation in the probability of recruitment between broods. Nonsignificant interactions $(P>0.3)$ were removed from the final model.

relationship between immune response and survival differed between unmanipulated and enlarged broods. Therefore, to explore this relationship further we performed additional analyses separately for unmanipulated and enlarged broods. Among unmanipulated broods the relationship between immune response and the probability of recruitment appeared significant (GLMM; $F_{1,253}=4.86, P<0.05$, Fig. 2$)$, while it was nonsignificant among enlarged broods (GLMM; $F_{1,215}=0.7$, n.s., Fig. 2 ). In both analyses the effect of body mass was not significant (GLMM; control broods: $F_{1,253}=0.12$, n.s.; enlarged broods: $F_{1,215}=$ 0.01 , n.s.). If body mass was removed from the

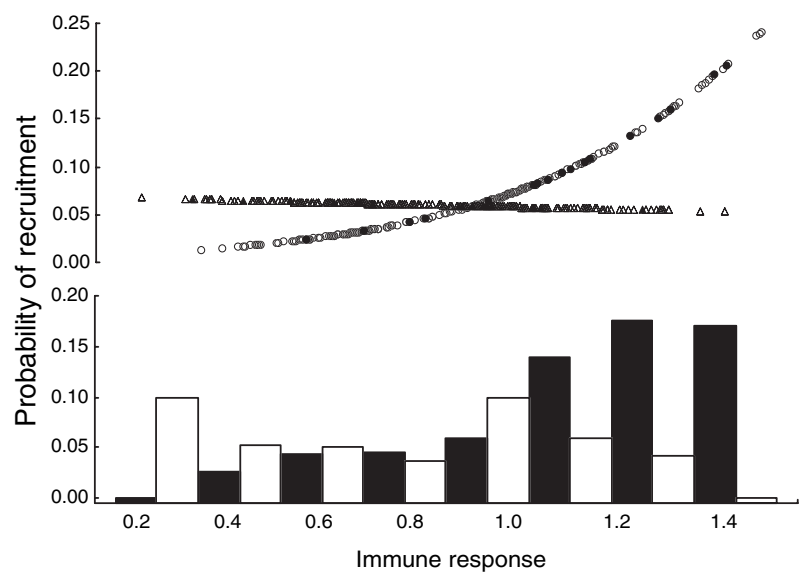

Fig. 2 The relationship between nestling immune response and the probability of local recruitment to the breeding population. Upper graphs present predicted probabilities derived from logistic regressions. Circles are individual nestlings from control broods, while triangles from enlarged broods. Filled circles and triangles represent surviving, while unfilled nonsurviving nestlings. The histogram shows proportions of surviving nestlings, black bars are proportions from control broods; white bars are proportions from enlarged broods. 
model the outcome was very similar (analyses not presented).

\section{Discussion}

This study shows that the probability of nestling survival until fledging, and, more importantly, the probability of being recruited to the local population in the following year, are positively related to nestling ability to respond to a novel antigen. Our results also show that the significant effect of immune response is independent of any potential effects of body mass. This is an important finding since body mass has repeatedly been reported to explain variation in survival probability in small passerine bird species (e.g. Tinbergen \& Boerlijst, 1990; Perrins $\delta$ McCleery, 2001) and body mass has also been shown to correlate with immune function (e.g. Tella et al., 2000a; Westneat et al., 2004). Therefore, any effects of immune function on survival could simply have stemmed from the above correlations. However, this turned out not to be the case. Thus, our results suggest that an ability to produce a strong immune response is indeed associated with higher viability. Positive correlation between immunocompetence and nestling survival until fledging has been reported for some free-living organisms (e.g. Nordling, 1998; González et al., 1999; Hõrak et al., 1999; Christe et al., 2001). Recent meta-analyses of survival in relation to some measures of immunocompetence across 12 studies of birds demonstrated relatively high mean effect size, suggesting that immune response is a reliable predictor of survival (Møller \& Saino, 2004). Evidence for the relationship between immunocompetence and longterm survival is reported in very few studies. Nestlings showing high immune response had higher chance of being recaptured a year later in the study area in collared flycatchers (Nordling, 1998) and house martin (Christe et al., 2001). Juvenile house sparrows showing higher immunocompetence had a greater probability of surviving during 3 months of outdoor housing and a higher probability of recovering from infection with blood parasites (González et al., 1999).

Our study shows that the relationship between T-cellmediated immune function and long-term survival is affected by rearing conditions. We found that this relationship was significant only among nestlings from control, unmanipulated broods, which had generally higher immune response and also higher probability of survival than nestlings from enlarged broods. This seems to be a very important finding, which can be interpreted in terms of larger developmental stress among nestlings from enlarged broods. Experimental brood enlargement typically results in retarded development of nestlings. It usually negatively affects 'nestlings' body size and the development of their immune system (e.g. Saino et al., 1997; Hõrak et al., 1999). In our study brood enlargement also resulted in suppression of T-cell-mediated immune response and lower body mass (unpublished data). We suggest that among enlarged broods, any effects of variation in immune function on survival probability may be overridden by other factors, which may have stronger effects in nestlings reared under poor environmental conditions. It is plausible that post-fledgling survival of nestlings from enlarged broods is more strongly influenced by retarded growth and development of some physiological functions rather than by retarded immune function. Body condition and development of flight feathers seem to be crucial determinants of an ability to avoid predators after reaching independence, while immune functions protect against pathogens. Survival is an outcome of the relative importance of these two sources of mortality. If retarded development makes individuals more susceptible to predation than to pathogens, the level of immune function may not predict survival.

The phenotypic variation in immune response has been shown to have a considerable genetic component (e.g. Saino et al., 1997; Brinkhof et al., 1999). Some studies, including the present one, have demonstrated a significant natural selection that may operate via differential survival in relation to immune function (see Møller \& Saino, 2004, for review). For example, Råberg et al. (2003) have shown in blue tit that antibody responsiveness may undergo either directional or stabilizing selection measured as differences in survival probability. Thus, basic requirements for selection to result in microevolutionary responses are fulfilled. However, benefits of high immune responsiveness in terms of increased survival may be counterbalanced if high immune responsiveness reduces fecundity. This seems plausible as immune function seems to trade for limited resources with reproduction (Deerenberg et al., 1997; Nordling et al., 1998; Cichoń et al., 2001). Microevolutionary responses may also be constrained, for example, by the existence of negative genetic correlations between immune function and some other vital traits, some phenotypic costs in terms of resource allocation trade-offs or if genes responsible for shaping immune function are expressed differently in different environments. These evolutionary constraints preventing depletion of genetic variation accompanied by the fact that immune responsiveness is condition-dependent may explain how the observed variation in immune function is maintained in natural populations. Our study suggests that selection for immune responsiveness seems to be weak or even absent under unfavourable rearing conditions, as survival was not related to immune response among nestlings from enlarged broods. Thus, yearly variation and environmental heterogeneity may constrain evolution towards higher immune responsiveness.

In conclusion, our results provide evidence for the important role played by immune defences in determining survival prospects in natural populations. We showed that long-term survival is positively related to the level of immune function only among control, unmanipulated broods and is not related to survival among nestlings 
from experimentally enlarged broods. We suggest that retarded development of nestlings from enlarged broods may to a larger extent increase mortality risk related to predation than risk of death from infection.

\section{Acknowledgments}

We thank Kinga Deptuch and Natalia Pitala for help in the field. Joanna Rutkowska provided valuable comments on earlier drafts of the manuscript. Michael Jacobs corrected the English. Financial support was provided by the State Committee for Scientific Research of Poland in 2004-2007.

\section{References}

Brinkhof, M.W.G., Heeb, P., Kolliker, M. \& Richner, H. 1999. Immunocompetence of nestling great tits in relation to rearing environment and parentage. Proc. R. Soc. Lond. B. 266: 1435 , 2315-2322.

Christe, P., De Lope, F., González, G., Saino, N. \& Møller, A.P. 2001. The influence of environmental conditions on immune responses, morphology and recapture probability of nestling house martins (Delichon urbica). Oecologia 126: 333-338.

Christe, P., Møller, A.P. \& De Lope, F. 1998. Immunocompetence and nestling survival in the house martin: the tasty chick hypothesis. Oikos 83: 175-179.

Cichoń, M., Dubiec, A. \& Chadzińska, M. 2001. The effect of elevated reproductive effort on humoral immune function in collared flycatcher females. Acta Oecol. 22: 71-76.

Deerenberg, C., Arpanius, V., Daan, S. \& Bos, N. 1997. Reproductive effort decreases antibody responsiveness. Proc. R. Soc. Lond. B. 264: 1021-1029.

Gershwin, M.E., Beach, R.S. \& Hurley, L.S. 1985. Nutrition and Immunity. Academic Press, Orlando.

González, G., Sorci, G., Møller, A.P., Ninni, P., Haussy, C. \& de Lope, F. 1999. Immunocompetence and condition-dependent sexual advertisement in male house sparrows (Passer domesticus). J. Anim. Ecol. 68: 1225-1234.

Goto, N., Kodama, H., Okada, K. \& Fujimoto, Y. 1978. Suppression of phytohemagglutinin skin response in thymectomized chickens. Poult. Sci. 52: 246-250.

Hõrak, P., Tegelmann, L., Ots, I. \& Møller, A.P. 1999. Immune function and survival of great tit nestlings in relation to growth conditions. Oecologia 121: 316-322.

Klasing, K.C. 1998. Nutritional modulation of resistance to infectious diseases. Poult. Sci. 77: 1119-1125.

Krackow, S. \& Tkadlec, E. 2001. Analysis of brood sex ratio: implication of offspring clustering. Behav. Ecol. Sociobiol. 50: 293-301.

Lessels, C.M. \& Boag, P.T. 1987. Unrepeatable repeatabilities: a common mistake. Auk 104: 116-121.

Lochmiller, R.L., Vestey, M.R. \& Boren, J.C. 1993. Relationship between protein nutritional status and immunocompetence in northern bobwhite chicks. Auk 110: 503-510.

Lochmiller, R.L. \& Deerenberg, C. 2000. Trade-offs in evolutionary immunology: just what is the cost of immunity? Oikos 88: $87-98$.

McGraw, K.J. \& Ardia, D.R. 2003. Carotenoids, immunocompetence, and the information content of sexual colors: an experimental test. Am. Nat. 162: 704-712.
Merilä, J., Przybylo, R., Sheldon, B.C. 1999. Genetic variation and natural selection on blue tit body condition in different environments. Genet. Res. 73: 165-176.

Møller, A.P. \& Saino, N. 2004. Immune response and survival. Oikos 104: 299-304.

Nordling, D., Andersson, M., Zohari, S. \& Gustafsson, L. 1998. Reproductive effort reduces specific immune response and parasite resistance. Proc. R. Soc. Lond. B. 265: 1291-1298.

Nordling, D. 1998. Trade-offs between life-history traits and immune defence in the collared flycatcher Ficedula albicollis. PhD Thesis, Uppsala University, Uppsala.

Norris, K. \& Evans, M.R. 2000. Ecological immunology: life history trade-offs and immune defense in birds. Behav. Ecol. 11: 19-26.

Pärt, T. \& Gustafsson, L. 1989. Breeding dispersal in the Collared Flycatcher (Ficedula albicollis) - possible causes and reproductive consequences. J. Anim. Ecol. 58: 305-320.

Perrins, C.M. \& McCleery, R.H. 2001. The effect of fledging mass on the lives of great tits Parus major. Ardea 89: 135-142.

Peters, A., Denk, A.G., Delhey, K. \& Kempenaers, B. 2004. Carotenoid-based bill colour as an indicator of immunocompetence and sperm performance in male mallards. J. Evol. Biol. 17: $1111-1120$.

Råberg, L. \& Stjernman, M. 2003. Natural selection on immune responsiveness in blue tits Parus caeruleus. Evoluion 57: 16701678.

Roitt, I., Brostoff, J. \& Male, D. 1996. Immunology. Mosby, London.

Saino, N., Calza, S. \& Møller, A.P. 1997. Immunocompetence of nestling barn swallows in relation to brood size and parental effort. J. Anim. Ecol. 66: 827-836.

Saino, N., Suffritti, C., Martinelli, R., Rubolini, D. \& Møller, A.P. 2003. Immune response covaries with corticosterone plasma levels under experimentally stressful conditions in nestling barn swallows (Hirundo rustica). Behav. Ecol. 14: 318-325.

SAS Institute, Inc. 2000. SAS/STAT User's Guide. Version 8.2. SAS Institute, Cary, NC.

Schmid-Hempel, P. 2003. Variation in immune defence as a question of evolutionary ecology. Proc. R. Soc. Lond. B. 270: 357-366.

Smits, J.E., Bortolotti, G.R. \& Tella, J.L. 1999. Simplifying the phytohaemagglutinin skin-testing technique in studies of avian immunocompetence. Funct. Ecol. 13: 567-572.

Tella, J.L., Bortolotti, G.R., Forero, M.G. \& Dawson R.D. 2000a. Environmental and genetic variation in T-cell-mediated immune response of fledgling American kestrels. Oecologia 123: 453-459.

Tella, J.L., Bortolotti, G.R., Dawson, R.D. \& Forero, M.G. 2000b. The T-cell-mediated immune response and return rate of fledgling American kestrels are positively correlated with parental clutch size. Proc. R. Soc. Lond. B. 267: 891-895.

Tinbergen, J.M. \& Boerlijst, M.C. 1990. Nestling weight and survival in individual great tits (Parus major). J. Anim. Ecol. 59: 1113-1127.

Westneat, D.F., Weiskittle, J., Edenfield, R., Kinnard, T.B. 8 Poston, J.P. 2004. Correlates of cell-mediated immunity in nestling house sparrows. Oecologia 141: 17-23.

Zuk, M. \& Stoehr, A.M. 2002. Immune defense and host life history. Am. Nat. 160: S9-S22.

Received 1 December 2004; revised 21 January 2005; accepted 25 January 2005 